\title{
Fingerprint Ridge Distance Estimation: Algorithms and the Performance*
}

\author{
Xiaosi Zhan ${ }^{1}$, Zhaocai Sun ${ }^{2}$, Yilong $\mathrm{Yin}^{2}$, and Yayun $\mathrm{Chu}^{1}$ \\ ${ }^{1}$ Computer Department, Fuyan Normal College, 236032, Fuyang, China \\ xiaoszhan@263.net, chuyayun. fync@163.com \\ ${ }^{2}$ School of Computer Science \& Technology, Shandong University, \\ 250100, Jinan, China \\ sunnykiller@126.com, ylyin@sdu.edu.cn
}

\begin{abstract}
Ridge distance is one important attribute of the fingerprint image and it also is one important parameter in the fingerprint enhancement. It is important for improving the AFIS's performance to estimate the ridge distance correctly. The paper discusses the representative fingerprint ridge distance estimation algorithms and the performance of these algorithms. The most common fingerprint ridge distance estimation algorithm is based on block-level and estimates the ridge distance by calculating the number of cycle pattern in the block fingerprint image. The traditional Fourier transform spectral analysis method has been also applied to estimate the fingerprint ridge distance. The next kind of method is based on the statistical window. Another novel fingerprint ridge distance estimation method is based on the region-level which regards the region with the consistent orientation as the statistical region. One new method obtains the fingerprint ridge distance from the continuous Fourier spectrum. After discussing the dominant algorithm thought, the paper analyzes the performance of each algorithm.
\end{abstract}

\section{Introduction}

The fingerprint images vary in quality. It is important to enhance effectively the fingerprint image with low quality for improving the performance of the automatic fingerprint identification system $[1,2,3]$. As one key attribute of the fingerprint image, most fingerprint enhancement algorithms regard the ridge distance as one essential parameter for enhancing the fingerprint image effectively. It is important to estimate the accurate ridge distance for improving the performance of the AFIS. In recent years, fingerprint ridge estimation method is the research focus and many methods for estimating the ridge distance have been brought forward in the correlative literatures.

D. C. Douglas Hung estimated the average distances of the all ridges on the whole fingerprint image [4]. Mario and Maltoni did mathematical characterization of the local frequency of sinusoidal signals and developed a 2-Dmodel of the ridge pattern in

* Supported by the National Natural Science Foundation of China under Grant No. 06403010, Shandong Province Science Foundation of China under Grant No.Z2004G05 and Anhui Province Education Department Science Foundation of China under Grant No.2005KJ089.

D. Zhang and A.K. Jain (Eds.): ICB 2006, LNCS 3832, pp. $294-301,2005$.

(C) Springer-Verlag Berlin Heidelberg 2005 
order to obtain the ridge density [5]. Lin and Dubes attempted to count ridge number in one fingerprint image automatically and assumed the ridge distance is a constant value on the whole fingerprint image [6]. L. Hong et al proposed the direction window method to estimate the ridge frequency [3]. O'Gorman and Nickerson acquired the ridge distance using the statistics mean value and used the ridge distance as a key parameter in the design of filters [7].Z. M. Kovace-Vajna et al brought out two kinds of ridge distance estimation methods: the geometry approach and the spectral analysis approach, which are both based on the block fingerprint image to estimate the ridge distance [8]. Y. Chen et al proposed two kinds of methods to estimate the ridge distance: the spectral analysis approach and the statistical window approach [9]. In addition, Y.L. Yin et al proposed the ridge distance estimation method based on regional level. The method divides the fingerprint image into several regions according to the consistency of the orientation information on the whole fingerprint image and calculates the ridge distance to every region respectively [10].

The paper chooses four representative fingerprint ridge estimation methods to analyze. After introducing the dominant realization steps, the paper analyzes the performance of the four methods mainly.

\section{The Primary Fingerprint Ridge Distance Algorithms}

Up to now, we can sum up the fingerprint ridge distance estimation algorithms into the following four primary kinds: (1) the statistical window method; (2) the regionlevel method; (3) the discrete Fourier spectral method and (4) the continuous spectrum analysis method.

\subsection{Method for Fingerprint Ridge Distance Estimation Based on Statistical Window}

The method defined the statistical window and the base line firstly. After dividing the fingerprint image into block image with the size of $32 \times 32$, the method estimated the ridge distance of each block image by detecting the distributing of the gray histogram. The definitions of the statistical window and the base line are showed as the following Fig. 1 and the key steps of the method can be described as:

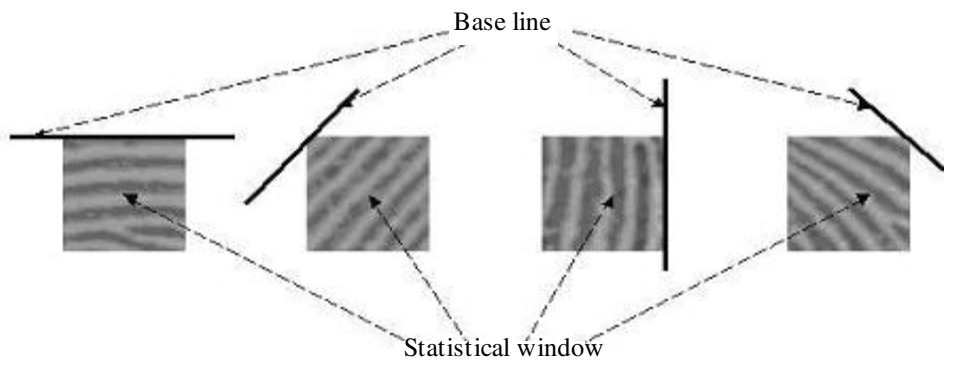

Fig. 1. Definitions of the statistical window and base line of different fingerprint image region 
Step 1: Calculate the fingerprint image orientation field based on block-level. Here, we can adopt the ridge orientation estimation method put forward by L. Hong et al. or other method.

Step 2: Translate the gray fingerprint image into the binary fingerprint image adopting the local self-adapt segmentation method.

Step 3: Define the base line and the statistical window of each block image according to the Fig.1. And then obtain the ridge distributing histogram in each block image.

Step 4: Detect and memorize the locality of all local peak value in the ridge distributing histogram. Obviously, every local peak value is corresponding to one ridge and the distance between the two adjacent points with peak value is the ridge distance between the two adjacent fingerprint ridges in the block image.

Step 5: Calculate the dependability degree of the ridge distance values in all fingerprint image region and adjust ridge distance value with low dependability degree.

\subsection{Method for Fingerprint Ridge Distance Estimation Based on Region Level}

The selecting of the window size is the key issue in the method in the statistical window method. It is the precondition for selecting the right window size to confirm the ridge distance. In theory, it is impossible. Consequently, Y. Long et al. put forward the method for estimating the fingerprint ridge distance based on the region level.

Based on the orientation field of block image, the method clustered the region with the close ridge orientation as one region by the region increasing method. Obviously, one fingerprint image can be segmented into several regions with the close ridge orientation. The following Fig. 2 showed the segmentation results:
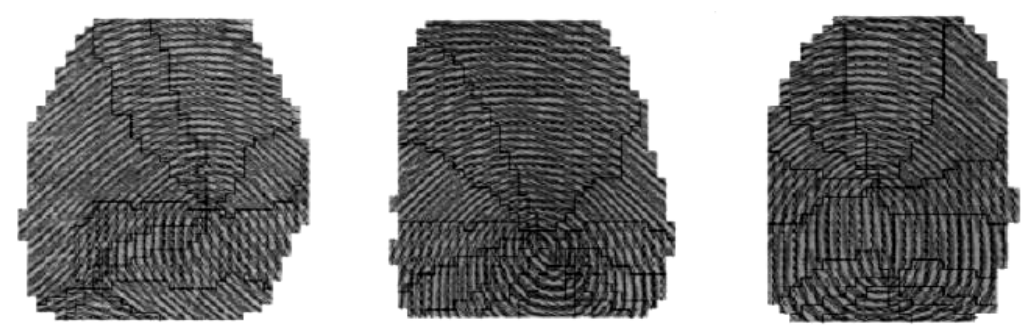

Fig. 2. Segmentation results of the directional images about three typical fingerprints

After segmenting the directional image into several regions, the method regarded every region with the same ridge orientation as one unit to estimate the ridge distance. The method can be described as the following steps.

Step 1: Calculate the area of each region. In the method, the region area can be defined as the number of the image blocks of the corresponding region. Go to step 2 if the number is more than or equal with the threshold value $R_{\min }\left(R_{\min }=8\right.$ in the paper).

Step 2: Define the statistical window and the base line of each region. Here, the statistical window and the base line is same with the definitions in 2.1. 
Step 3: Translate the gray fingerprint image into the binary fingerprint image adopting the local self-adapt segmentation method.

Step 4: Calculate and memorize the distance between the ridge pixel in the region and the base line. Obtain the ridge distributing histogram on the reference frame by defining the distance to the base line and the number of the ridge pixel in the same distance as the x-axle and $y$-axle respectively.

Step 5: Estimate the ridge distance in one region by obtaining the peak values of the corresponding histogram.

Step 6: The ridge distance of one region with the area smaller than Rmin can be defined the average value of the ridge distances in the circumjacent regions.

\subsection{Fingerprint Ridge Distance Estimation Based on the Discrete Fourier Spectrum}

Spectral analysis method that transforms the representation of fingerprint images from spatial field to frequency field is a typical method of signal processing in frequency field. It is a traditional method for ridge distance estimation in fingerprint images. Generally, if $g(x, y)$ is the gray-scale value of the pixel $(\mathrm{x}, \mathrm{y})$ in an $N \times N$ image, the DFT of the $g(x, y)$ is defined as follows:

$$
G(u, v)=\frac{1}{N^{2}} \sum_{i=1}^{N} \sum_{j=1}^{N} g(x, y) e^{2 \pi j / N<(x, y)(u, v)>}
$$

Where $\mathrm{j}$ is the imaginary unit, $u, v \in\{1, \cdots N\}$ and $\langle(x, y)(u, v)\rangle=x u+y v$ is the vector pot product. In theory, the module $\left|G_{(u, v)}\right|$ of $G(u, v)$ describes the cycle character of signal. We can acquire the dominant cycle of the signal in one region by calculating the values of $\left|G_{(u, v)}\right|$ of each pixel in the region, which can be defined the ridge frequency in the fingerprint ridge distance estimation. For obtaining the right ridge distance value, Y. Chen et al. define the radial distribute function in [10] as follows:

$$
Q(r)=\frac{1}{\# C_{r}} \sum_{(u, v) \in C_{r}}\left|G_{(u, v)}\right|
$$

Where $C_{r}$ is the set of the whole pixels that satisfy the function $\sqrt{u^{2}+v^{2}}=r$, $\# \boldsymbol{C}_{\boldsymbol{r}}$ is the number of the element of the set $C_{r}$. Then define the $\boldsymbol{Q}(\boldsymbol{r})$ as the distribution intensity of the signal with the cycle $N / r$ in the $N \times N$ image. The value of $r$ corresponding to the peak of $\boldsymbol{Q}(\boldsymbol{r})$ can be defined as the cycle number of the dominant signal in the $N \times N$ image. Search for the value of $r_{0}$ that enables the value of $\boldsymbol{Q}\left(\boldsymbol{r}_{\mathbf{0}}\right)$ is the local maximum. At this moment, the ridge distance of the block image can be estimated with $d=N / r_{0}$. The dominant steps of the method can be described as the following:

Step 1: Divide the fingerprint image into non-overlap block image with the $\mathrm{N} \times \mathrm{N}$ size ( $\mathrm{N}$ is 32 generally).

Step 2: Calculate $\left|\boldsymbol{G}_{(\boldsymbol{u}, \boldsymbol{v})}\right|$ of each pixel $(x, y)(x, y \in\{0, \cdots, 31\})$ in one block image by adopting the 2-D fast Fourier transform. 
Step 3: Calculate the value of $Q(r)(0 \leq r \leq N-1)$.

Step 4: Search the appropriate $r^{\prime}$ which make each $r\left(\mathbf{0} \leq \boldsymbol{r}_{\min } \leq \boldsymbol{r} \leq \boldsymbol{r}_{\max } \leq \boldsymbol{N}-\mathbf{1}\right.$ and $\left.\boldsymbol{r} \neq \boldsymbol{r}^{\prime}\right)$ having $\boldsymbol{Q}\left(\boldsymbol{r}^{\prime}\right) \geq \boldsymbol{Q}(\boldsymbol{r})$.

Step 5: Cannot estimate the ridge distance of one block image if the following two conditions cannot be satisfied: $\boldsymbol{Q}\left(\boldsymbol{r}^{\prime}\right)>\boldsymbol{Q}(\boldsymbol{r}-\mathbf{1})$ and $\boldsymbol{Q}\left(\boldsymbol{r}^{\prime}\right)>\boldsymbol{Q}(\boldsymbol{r}+\mathbf{1})$. Here, we think that $\boldsymbol{Q}\left(\boldsymbol{r}^{\prime}\right)$ is not the local peak value. Otherwise, search the appropriate $r^{\prime \prime}$ that makes each $\boldsymbol{r}\left(\boldsymbol{0} \leq \boldsymbol{r}_{\min } \leq \boldsymbol{r} \leq \boldsymbol{r}_{\max } \leq \boldsymbol{N}-\mathbf{1}, \boldsymbol{r} \neq \boldsymbol{r}^{\prime}\right.$ and $\left.\boldsymbol{r} \neq \boldsymbol{r}^{\prime \prime}\right)$ having $\boldsymbol{Q}\left(\boldsymbol{r}^{\prime \prime}\right) \geq \boldsymbol{Q}(\boldsymbol{r})$.

Step 6: Calculate the dependability degree according to the following formula:

$$
\frac{\alpha}{\boldsymbol{Q}\left(\boldsymbol{r}^{\prime}\right)} \min \left\{\boldsymbol{Q}\left(\boldsymbol{r}^{\prime}\right)-\boldsymbol{Q}\left(\boldsymbol{r}^{\prime \prime}\right), \boldsymbol{Q}\left(\boldsymbol{r}^{\prime}\right)-\boldsymbol{Q}\left(\boldsymbol{r}^{\prime}-\mathbf{1}\right), \boldsymbol{Q}\left(\boldsymbol{r}^{\prime}\right)-\boldsymbol{Q}\left(\boldsymbol{r}^{\prime}+\mathbf{1}\right)\right\}
$$

Estimate the ridge distance of one block image with the formula $\bar{d}=N / \boldsymbol{r}$ when the dependability degree is larger than 0.4 . Otherwise, the ridge distance of one block image can' t be estimated.

\subsection{Ridge Distance Estimation Method Based on the Continues Spectrum}

The precision does not meet the requirement if we carry through the discrete Fourier transform. At the same time, the speed can't meet the requirement of the real time disposal if we make the continuous Fourier transform. So, the method adopts the 2-D sampling theorem to transform the 2-D discrete Fourier spectrum into 2-D continuous Fourier spectrum and estimates the ridge distance based on the continuous spectrum.

Suppose the Fourier transform function $F\left(s_{1}, s_{2}\right)$ about the function $\mathrm{f}(\mathrm{x} 1, \mathrm{x} 2)$ of L2 (R2) is tight-supported set (namely that the function $F$ is equal to zero except the boundary region $\mathrm{D}$ and the boundary region $\mathrm{D}$ can be defined as the rectangle region $\left\{\left(s_{1}, s_{2}\right)|| s_{1} \mid \leq \Omega\right.$ and $\left.\left|s_{2}\right| \leq \Omega\right\}$ in the paper. Here, we firstly assume $\Omega=\pi$ in order to simplify the function. Then the Fourier transform function about the function $\mathrm{f}\left(\mathrm{x}_{1}, \mathrm{x}_{2}\right)$ can be denoted as follows:

$$
F\left(s_{1}, s_{2}\right)=\sum_{n_{1}} \sum_{n_{2}} C_{n_{1}, n_{2}} e^{-j n_{1} s_{1}-j n_{2} s_{2}}
$$

Here, the $C_{n_{1}, n_{2}}$ is defined as follows:

$$
C_{n_{1}, n_{2}}=\frac{1}{(2 \pi)^{2}} \int_{-\infty}^{+\infty} \int_{-\infty}^{+\infty} d s_{1} d s_{2} e^{j n_{1} s_{1}+j n_{2} s_{2}} F\left(s_{1}, s_{2}\right)=\frac{1}{2 \pi} f\left(n_{1}, n_{2}\right)
$$

Then, we can acquire the following function as:

$$
f\left(x_{1}, x_{2}\right)=\sum_{n_{1}} \sum_{n_{2}} C_{n_{1}, n_{2}} \frac{\sin \pi\left(x_{1}-n_{1}\right)}{\pi\left(x_{1}-n_{1}\right)} \frac{\sin \pi\left(x_{2}-n_{2}\right)}{\pi\left(x_{2}-n_{2}\right)}
$$

In this way, the discrete signal $\mathrm{C}_{\mathrm{n} 1},{ }_{\mathrm{n} 2}$ can be recovered for the continuous signal $f\left(x_{1}, x_{2}\right)$ through the sampling theorem. Then, the discrete frequency spectrum of each block fingerprint image can be recovered for the continuous frequency spectrum. 


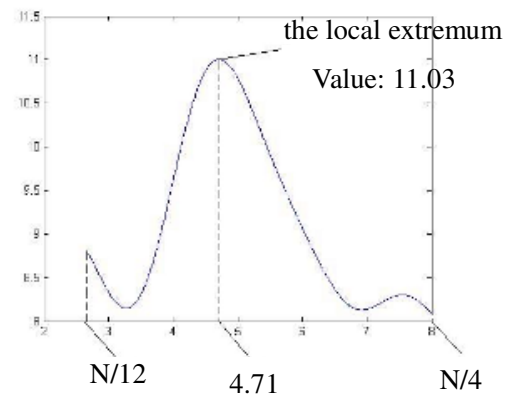

Fig. 3. The cutaway view of the continuous spectrum in the normal orientation

We can try to obtain accurately the local extreme value (that is the "light spot" position we care about) in random small step in the continuous frequency spectrum. Thus we can calculate the ridge distance accurately. But it is a long period course that we search the continuous spectrum image that is recovered from one $N \times \boldsymbol{N}$ point matrix in a small step for the local peak value. Thus we need to search the continuous spectrum purposefully.

Suppose that the ridge orientation is $\theta$ and then the normal orientation of the ridge is $\theta+\pi / 2$. We can obtain the position of the local extreme point in the continuous spectrum if we search the region, which is confirmed by the radius: N/12-N/4 and the direction: $\theta+\pi / 2$, in the step length as 0.01 . As Fig.3 shows, the local extreme points are 11.03 , the corresponding radius is 4.71 , and the ridge distance of the image is $32 / 4.71=6.79$.

Step 1: Divide the fingerprint image into non-overlap block with the size $N \times N$, the $\mathrm{N}$ is equal to 32 generally.

Step 2: To each block fingerprint image $\boldsymbol{g}(\boldsymbol{i}, \boldsymbol{j})$, carry on two-dimension fast Fourier transform and get the corresponding discrete spectrum $G(u, v)$.

Step 3: To each discrete spectrum $_{G(u, v)}$, apply the sampling theorem to get the continuous spectral function $\mathrm{G}(\mathrm{x}, \mathrm{y})$.

Step 4: Adopt Rao method to obtain the ridge orientation $\theta$.

Step 5: Search the region confirmed by the radius N/12-N/4 and the direction $\theta+\pi / 2$ in a small step length $\mathrm{L}$ for finding the radius $\mathrm{r}$ corresponding the local extreme point. Generally, the value of $\mathrm{L}$ is 0.01 .

Step 6: If don't find the local extreme point then think that the ridge distance of the fingerprint image region can't be obtained. Else estimate the fingerprint image ridge distance from $\mathrm{d}=\mathrm{N} / \mathrm{r}$.

\section{Performance Analysis and Conclusion}

To evaluate the performance of the methods, we use 30 typical images (10 good quality, 10 fair quality, 10 poor quality) selected from NJU fingerprint database (1200 livescan images; 10 per individual) to estimate ridge distance with the four ridge distance estimation methods respectively. In order to describe the performance in the same 
criterions, the paper chooses the following three criterions DER, EA and TC. Here, DER indicates the robustness of a method for ridge distance estimation in fingerprint images, EA is the degree of deviation between the estimation result and the actual value of the ridge distance and TC is the time needed for handing a fingerprint image. A high DER value means that the method is flexible and insensitive to a variety of image quality and ridge directions. A high EA value indicates that the estimation result is close to the actual value of the ridge distance. A lower TC value means that the method is faster. The following table 1 is the performance of the four methods.

From the Table 1, we can obtain the following conclusion. (1) The statistical method has the middle DER value, the EA value and the TC value. For the method, the sixty-four dollar question is that the method cannot estimate the ridge distance in a good deal of region. It doesn't perform well in these regions where there is acute variation of ridge directions. But, the obvious advantage of the statistical window method is that it is simple and can estimate the right ridge distance in good quality image region. (2) The region-level method has the highest DER value with the lowest EA and TC values. The method divides the fingerprint image into several regions and the ridge distance of each region can be estimated generally. But, the ridge distance is not accurate in most block images because there is only one ridge distance value in one big region and the ridge distance is diverse in the same region. (3) The discrete spectrum method has the lowest DER value with the middle TC value and EA value. For this method, the biggest problem is how to determine e r' accurately and reliably. If we can acquire the value of $r^{\prime}$ accurately and reliably, the performance will improve significantly. (4) The continuous spectrum has the highest EA value and TC value with the middle DER value. The method can obtain the ridge distance of most regions in a fingerprint image except the pattern region and the strong-disturbed region because the sub-peak is not obvious in these regions. In addition, the processing time of our method is more that the other two methods because our method is based on the two-dimension continuous spectrum. It shows that the method has higher performance except the processing time.

In order to illustrate the performance of the four methods farther, the paper chooses 10 representative fingerprint images ( 5 fingerprint images with good quality, 3 fingerprint images with fair quality and 2 fingerprint images with low quality) to test the effect on the minutiae exactness method. Firstly, we extract the right minutia artificially and consider the minutia set as the standard minutia set. Then, we extract the minutiae with the same processing methods except the ridge estimation method. Here, we define TMN, RMN, LMN, EMN and Rate as the total minutiae number, the lost minutiae number, the right minutiae number, the error minutiae number and the accurate rate respectively. Here, we define the accurate rate as the ratio between the RMN and the sum of the TMN and the LMN. The test results is showed as the following Table.2:

Table 1. The three performance indexes of the four methods

\begin{tabular}{cccc}
\hline Method & DER $(\%)$ & EA $(\%)$ & TC (second) \\
\hline Statistical window method & 63.8 & 93 & 0.31 \\
Region-level method & 100 & 68 & 0.28 \\
Discrete spectrum method & 44.7 & 84 & 0.42 \\
Continuous spectrum method & 94.6 & 95 & 0.63 \\
\hline
\end{tabular}


Table 2. The minutiae exactness results of the four methods

\begin{tabular}{cccccc}
\hline Method & TMN & LMN & RMN & EMN & Rate (\%) \\
\hline Statistical window method & 484 & 32 & 448 & 36 & 86.8 \\
Region-level method & 512 & 26 & 440 & 72 & 81.8 \\
Discrete spectrum method & 501 & 24 & 445 & 56 & 84.8 \\
Continuous spectrum method & 487 & 15 & 459 & 28 & 91.4 \\
\hline
\end{tabular}

From Table 2 we can find that the continuous spectrum method has the highest performance with the lowest LMN EMN values and the highest RMN and Rate values. For region-level method, the processing result is affected by the fingerprint image quality. The method can't process well for these fingerprint images with low quality. Generally, the statistical window method can process well except some strive noised fingerprint images.

For the fingerprint ridge estimation, we should combine the availability of the methods based on spatial field and frequency field. Continuous spectrum analysis method has its merits and has the highest performance. But, the key issues are that the time consuming is very high and we should search better method for transforming the spatial fingerprint image into two-dimension continuous frequency spectrum and making certain the more appropriate step length in order to find the two sub-peak points faster and accurately.

\section{References}

[1] L. Hong, A.K. Jain, R. Bolle et al. Identity authentication using fingerprints. Proceedings of FirstInternational Conference on Audio and Video-Based Biometric Person Authentication, Switzerland, 1997:103-110.

[2] L. Yin, X. Ning, X. Zhang. Development and application of automatic fingerprint identification technology.Journal of Nanjing University(Natural Science), 2002, 38(1):29-35.

[3] L. Hong, Y. Wan, A. K. Jain. Fingerprint image enhancement: algorithm and performance evaluation, IEEE Transactions on Pattern Analysis and Machine Intelligence, 1998, 20(8): 777-789.

[4] D. C. Douglas Hung, Enhancement feature purification of fingerprint images, Pattern Recognition, 1993, 26(11): 1661-1671.

[5] D. Maio and D. Maltoni. Ridge-line density estimation in digital images. Proceedings of 14th International Conference on Pattern Recognition, Brisbane, Australia, 1998: 534538.

[6] W. C. Lin and R. C. Dubes. A review of ridge counting in dermatoglyphics, Pattern Recognition, 1983, 16(2): 1-8.

[7] L. O'Gorman, J. V. Neckerson. An approach to fingerprint filter design, Pattern Recognition, 1989, 22(1): 29-38.

[8] Z. M. Kovacs-Vajna, R. Rovatti, and M. Frazzoni, Fingerprint ridge distance computation methodologies, Pattern Recognition, 33 (2000) 69-80.

[9] Y. Chen, Y. Yin, X. Zhang et al, A method based on statistics window for ridge distance estimation, Journal of image and graphics, China, 2003, 8(3): 266-270.

[10] Y. Yin, Y Wang, F Yu, A method based on region level for ridge distance estimation, Chinese computer science, 2003, 30(5): 201-208. 